\title{
MINIMAL PERIODIC ORBITS OF CONTINUOUS MAPPINGS OF THE CIRCLE
}

\author{
JAUME LLIBRE
}

\begin{abstract}
Let $f$ be a continuous map of the circle into itself and suppose that $n>1$ is the least integer which occurs as a period of a periodic orbit of $f$. Then we say that a periodic orbit $\left\{p_{1}, \ldots, p_{n}\right\}$ is minimal if its period is $n$. We classify the minimal periodic orbits, that is, we describe how the map $f$ must act on the minimal periodic orbits. We show that there are $\varphi(n)$ types of minimal periodic orbits of period $n$, where $\varphi$ is the Euler phi-function.
\end{abstract}

1. Introduction and statement of results. Let $C(X, X)$ denote the set of continuous maps of a space $X$ into itself. A point $p \in X$ is a periodic point of a map $f \in C(X, X)$ if $f^{n}(p)=p$ for some positive integer $n$. The period of $p$ is the least such integer $n$, and the orbit of $p$ is the set $\left\{f^{k}(p): k=1, \ldots, n\right\}$. We refer to such an orbit as a periodic orbit of period $n$.

Let $P(f)$ denote the set of positive integers $n$ such that $f$ has a periodic point of period $n$. The following theorem for periodic orbits of maps of the closed interval $I$ is proved in [5] (see also [3]).

TheOREM (ŠTEFAN). Let $f \in C(I, I)$. Suppose $n \in P(f)$ where $n$ is odd and $n>1$, but $j \notin P(f)$ for all $j \in\{3,5, \ldots, n-2\}$. Let $\left\{p_{1}, \ldots, p_{n}\right\}$ be a periodic orbit of $f$ of period $n$ with $p_{1}<p_{2}<\cdots<p_{n}$. Let $t=(n+1) / 2$. Then either (A) or (B) holds:

$$
\begin{aligned}
f\left(p_{t-k}\right) & =p_{t+k} & & \text { for } k=1, \ldots, t-1, \\
f\left(p_{t+k}\right) & =p_{t-k-1} & & \text { for } k=0, \ldots, t-2, \quad \text { and } \\
f\left(p_{n}\right) & =p_{t} . & & \\
f\left(p_{t-k}\right) & =p_{t+k+1} & & \text { for } k=0, \ldots, t-2, \\
f\left(p_{t+k}\right) & =p_{t-k} & & \text { for } k=1, \ldots, t-1, \quad \text { and } \\
f\left(p_{1}\right) & =p_{t} . & &
\end{aligned}
$$

In this paper we obtain a similar result for periodic orbits of maps of the circle $S^{1}$. For distinct points $a, b \in S^{1}$, let $(a, b)$ and $[a, b]$ denote the open and closed intervals, respectively, from $a$ counterclockwise to $b$.

Theorem A. Let $f \in C\left(S^{1}, S^{1}\right)$. Suppose $n \in P(f)$ where $n>1$, and $j \notin P(f)$ for all $j \in\{1,2, \ldots, n-1\}$. Let $P=\left\{p_{1}, \ldots, p_{n}\right\}$ be a periodic orbit of $f$ of period $n$

Received by the editors November 4, 1980.

1980 Mathematics Subject Classification. Primary 54H20. 
where $P \cap\left(p_{k}, p_{k+1}\right)=\varnothing$ for $k=1, \ldots, n-1$ and $P \cap\left(p_{n}, p_{1}\right)=\varnothing$. Then $f\left(p_{k}\right)$ $=p_{\sigma(k)}$, where $\sigma=\tau^{t}$ with $1 \leqslant t<n, t$ relatively prime to $n$, and $\tau$ is the permutation of $\{1,2, \ldots, n\}$ given by $\tau(k)=k+1$ for $k=1, \ldots, n-1$ and $\tau(n)=1$.

This theorem will be proved in $\$ 3$.

We remark that, in Theorem A, $n$ is the smallest element of $P(f)$. Then we say that a periodic orbit $P$ is minimal if its period is $n$. Therefore, Theorem $A$ describes how a mapping $f \in C\left(S^{1}, S^{1}\right)$ must act on a minimal periodic orbit. Furthermore, by Theorem $\mathrm{A}$, there are $\varphi(n)$ types of minimal periodic orbits of period $n$, where $\varphi$ is the Euler phi-function.

Let $R$ be the real line, $C$ the complex numbers, and take $S^{1}=\{z \in C:|z|=1\}$. We use the universal covering $E \in C\left(R, S^{1}\right)$ given by $E(x)=e^{2 \pi i x}$. Let $f \in$ $C\left(S^{1}, S^{1}\right)$, and let $F \in C(R, R)$ be a lifting of $f$ to the covering space. If $F$ and $F^{\prime}$ are liftings of the same map $f$, then $F=F^{\prime}+k$ for some integer $k$. There exists an integer $N$ (the degree of $f$ ) such that $F(x+1)=F(x)+N$ for all $x$.

The following lemma is well known. For a proof see [4, p. 107].

Lemma 1. Let $f \in C\left(S^{1}, S^{1}\right)$ and let $N$ be the degree of $f$. Then $f$ has at least $|1-N|$ fixed points.

From this lemma, if $f \in C\left(S^{1}, S^{1}\right)$ has minimal periodic orbits of period $n$ with $n>1$, then the degree of $f$ is 1 .

Let $f \in C\left(S^{1}, S^{1}\right)$ and suppose the degree of $f$ is 1 . Fix a lifting $F$ of $f$. If $p$ is a periodic point of $f$ of period $n$ and $E(x)=p$, then $F^{n}(x)=x+k$ for some integer $k$. We shall call the number $k / n$ the rotation number of $p$ and denote it by $\rho_{F}(p)$. It is easy to see that $\rho_{F}(p)$ does not depend on the choice of $x$, and that if $F^{\prime}=F+m$ then $\rho_{F^{\prime}}(p)=\rho_{F}(p)+m$. For more details on the rotation number see [2].

From Theorem A, it is immediate to prove the following.

COROLlary B. In the hypotheses of Theorem A, let $p_{i}$ be a periodic point of the minimal periodic orbit $P$, and let $F$ be a lifting of $f$ such that $F(x) \in[0,1)$ where $E(x)=p_{i}$. Then we have $\rho_{F}\left(p_{i}\right)=t / n$.

Note that each minimal periodic orbit is realizable for a suitable rotation map of the circle.

2. Preliminary results. Let $I$ and $J$ be proper closed intervals on $S^{1}$ and let $f \in C\left(S^{1}, S^{1}\right)$. We say $I f$-covers $J$ if, for some closed interval $K \subset I, f(K)=J$.

We state the following three lemmas of Block, which will be used in the next section.

LEMMA 2 (LEMMA 1 OF [1]). Let $I=[a, b]$ be a proper closed interval on $S^{1}$ and let $f \in C\left(S^{1}, S^{1}\right)$. Suppose $f(a)=c$ and $f(b)=d$ and $c \neq d$. Then either $I f$-covers $[c, d]$ or I f-covers $[d, c]$.

LeMMa 3 (LemMa 2 of [1]). Let $f \in C\left(S^{1}, S^{1}\right)$. Let $I$ and $J$ be proper closed intervals on $S^{1}$ such that $I f$-covers $J$. Suppose $L$ is a closed interval with $L \subset J$. Then I f-covers $L$. 
LemMa 4 (Lemma 3 of [1]). Let $f \in C\left(S^{1}, S^{1}\right)$. Suppose $N$ is a proper closed interval on $S^{1}$ such that $N$-covers $N$. Then $f$ has a fixed point in $N$.

3. Proof of Theorem A. Let $f \in C\left(S^{1}, S^{1}\right)$ and suppose that $n>1$ is the smallest element of $P(f)$. Let $P=\left\{p_{1}, \ldots, p_{n}\right\}$ be a periodic orbit of $f$ of period $n$ where $P \cap\left(p_{k}, p_{k+1}\right)=\varnothing$ for $k=1, \ldots, n-1$ and $P \cap\left(p_{n}, p_{1}\right)=\varnothing$. Finally, we let $I_{k}=\left[p_{k}, p_{k+1}\right]$ for $k=1, \ldots, n-1$ and $I_{n}=\left[p_{n}, p_{1}\right]$.

For $n=2$ or $n=3$, Theorem $A$ is immediate. Then we may assume that $n \geqslant 4$.

By Lemma 4, $I_{k}$ does not $f$-cover $I_{k}$ for all $k=1, \ldots, n$. From Lemmas 2 and 3, $I_{k} f$-covers $I_{j}$ for some $j \neq k$ and for all $k=1, \ldots, n$. Hence for some set of distinct $I_{k}$ 's, $\left\{I_{k_{1}}, \ldots, I_{k_{m}}\right\}, I_{k_{i}} f$-covers $I_{k_{i+1}}$ for $i=1, \ldots, m-1$ and $I_{k_{m}} f$-covers $I_{k_{1}}$, where $2 \leqslant m \leqslant n$.

Since $I_{k_{m}} f$-covers $I_{k_{1}}$, there is a closed interval $J_{m} \subset I_{k_{m}}$ such that $f\left(J_{m}\right)=I_{k_{1}}$. Similarly, there are closed intervals $J_{1}, \ldots, J_{m-1}$ such that, for $i=1, \ldots, m-1$, $J_{i} \subset I_{k_{i}}$ and $f\left(J_{i}\right)=J_{i+1}$. It follows that $f^{m}\left(J_{1}\right)=I_{k_{1}}$. By Lemma $4, f^{m}$ has a fixed point in $I_{k_{1}}$. Then $m=n$. This implies that each $I_{k} f$-covers only one $I_{j}$, for some $j \neq k$. Therefore, $P \cap f\left(I_{k}\right)=\left\{f\left(p_{k}\right), f\left(p_{k+1}\right)\right\}$ for $k=1, \ldots, n-1$ and $P \cap$ $f\left(I_{n}\right)=\left\{f\left(p_{1}\right), f\left(p_{n}\right)\right\}$.

In particular, we have that $I_{1} f$-covers $I_{j}$ for some $j \neq 1$. Suppose the following is true:

$$
I_{j}=\left[f\left(p_{2}\right), f\left(p_{1}\right)\right]
$$

Therefore $j \neq n$. Since each $I_{k} f$-covers only one $I_{i}$, for some $i \neq k$, by continuity we have that $f\left(p_{j / 2+1}\right) \in\left\{p_{j / 2+1}, p_{j / 2+3}\right\}$ if $j$ is even (see Figure 1). But this is a contradiction, because $f\left(p_{j / 2+1}\right) \neq p_{j / 2+1}$ and $f\left(p_{j / 2-1}\right)=p_{j / 2+3}$. If $j$ is odd, then we have that $f\left(p_{(j+3) / 2}\right) \in\left\{p_{(j+1) / 2}, p_{(j+5) / 2}\right\}$ (see Figure 2). Again, this is a contradiction, since $f\left(p_{(j+1) / 2}\right)=p_{(j+3) / 2}$ and $f\left(p_{(j-1) / 2}\right)=p_{(j+5) / 2}$.

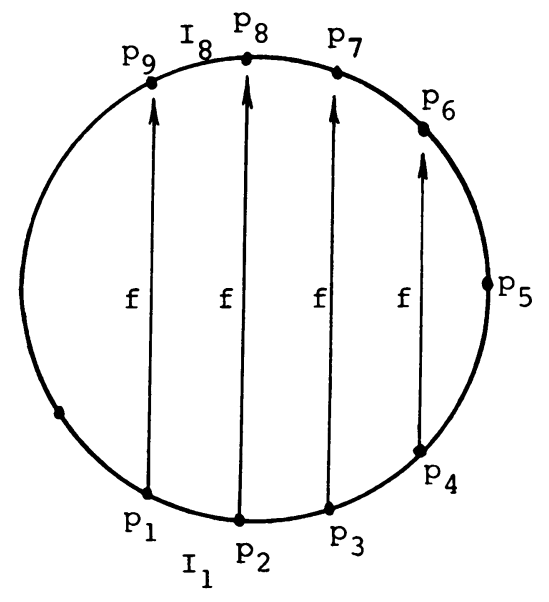

Figure 1 


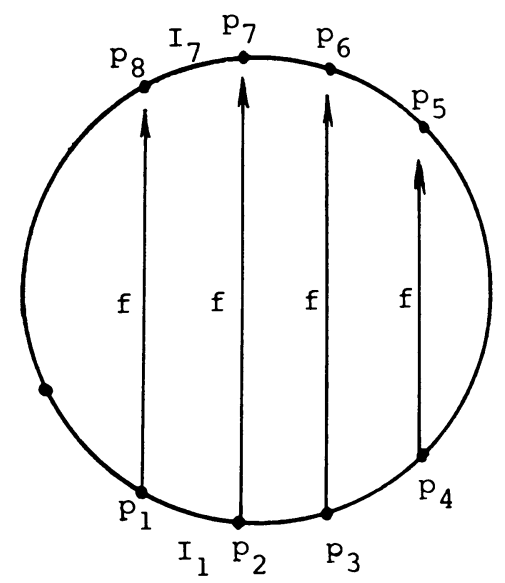

FIGURE 2

Thus the following must be true:

$$
I_{j}=\left[f\left(p_{1}\right), f\left(p_{2}\right)\right] .
$$

Then $f\left(p_{1}\right)=p_{j}, f\left(p_{2}\right)=p_{j+1}$ if $j \neq n$ or $f\left(p_{2}\right)=p_{1}$ if $j=n$. Since each $I_{k}$ $f$-covers only one $I_{i}$, for some $i \neq k$, by continuity we have that there exists $t \in\{1,2, \ldots, n-1\}$ such that $f\left(p_{k}\right)=p_{o(k)}$ where $\sigma$ is a permutation of $\{1,2, \ldots, n\}$ such that:

(a) $\sigma(k)=k+t$ if $k+t \leqslant n$, and $\sigma(k)=k+t-n$ if $k+t>n$.

(b) $\sigma^{i}$ is not the identity for all $i \in\{1,2, \ldots, n-1\}$. Then Theorem A follows.

\section{REFERENCES}

1. L. Block, Periodic orbits of continuous mappings of the circle, Trans. Amer. Math. Soc. 260 (1980), 553-562.

2. L. Block, J. Guckenheimer, M. Misiurewicz and L. S. Young, Periodic points and topological entropy of one dimensional maps, Proc. Conf. Global Theory of Dynamical Systems (Northwestern University) (Z. Nitecki and C. Robinson, eds.), Lecture Notes in Math., vol. 819, Springer-Verlag, Berlin and New York, 1980, pp. 18-34.

3. L. Block, Stability of periodic orbits in the theorem of Šarkooskii, Proc. Amer. Math. Soc. 81 (1981), 333-337.

4. R. F. Brown, The Lefschetz fixed point theorem, Scott, Foresman \& Co., Glenview, Ill., 1971.

5. P. Stefan, $A$ theorem of Sarkouskii on the existence of periodic orbits of continuous endomorphisms of the real line, Comm. Math. Phys. 54 (1977), 237-248.

Secció de Matemàttques, facultat de Ciències, Universitat Autònoma de Barcelona, BelLATERra, Barcelona, Spain 